\title{
MicroRNA-148a is silenced by hypermethylation and interacts with DNA methyltransferase 1 in hepatocellular carcinogenesis
}

\author{
XIAO-RAN LONG ${ }^{1-3}$, YONG HE ${ }^{1-3}$, CHENG HUANG ${ }^{1-3}$ and JUN LI ${ }^{1-3}$ \\ ${ }^{1}$ School of Pharmacy, Anhui Key Laboratory of Bioactivity of Natural Products, Anhui Medical University; \\ ${ }^{2}$ The Key Laboratory of Anti-Inflammatory and Immune Medicine (Anhui Medical University), Ministry of Education; \\ ${ }^{3}$ Institute for Liver Diseases of Anhui Medical University, Anhui Medical University, P.R. China
}

Received November 30, 2013; Accepted January 13, 2014

DOI: 10.3892/ijo.2014.2373

\begin{abstract}
In general, microRNAs, a class of small ( 21 nucleotide) non-coding RNAs, negatively regulate the expression of their target genes. Dysregulation of miRNAs is a common feature in human cancers, but this phenomenon has not been studied extensively in hepatocellular carcinoma (HCC). miR-148a, a member of the miR-148/152 family, has been found to be downregulated in several tumor types and has been suggested to be a tumor suppressor gene; however, its function in HCC remains unclear. Herein, we describe the epigenetic regulation of miR-148a and its impact on HCC cells. We found that, due to the hypermethylation of its $\mathrm{CpG}$ island, miR-148a undergoes methylation-mediated silencing in HCC cell lines. Additionally, DNMT1, the DNA methyltransferase that maintains methylation patterns, is aberrantly upregulated in HCC cell lines, and its overexpression is responsible for hypermethylation of the miR-148a promoter. Intriguingly, the expression of DNMT1, which is a target of miR-148a, is inversely correlated with the expression of miR-148a in HCC cells. These results lead us to propose the existence of a negative feedback regulatory loop between miR-148a and DNMT1 in HCC. Importantly, we demonstrate that the overexpression of miR-148a significantly inhibits HCC cell proliferation and cell cycle progression. Our results suggest the existence of a novel miR-148a-DNMT1 regulatory circuit and indicate
\end{abstract}

Correspondence to: Professor Jun Li, School of Pharmacy, Anhui Medical University, Mei Shan Road, Hefei 230032, P.R. China E-mail: 1j@ahmu.edu.cn

Abbreviations: HCC, hepatocellular carcinoma; miRNA, microRNA; DNMT1, DNA methyltransferase 1; RT-PCR, semi-quantitative reverse transcription-polymerase chain reaction; RT-qPCR, quantitative real-time PCR; PBS, phosphate-buffered saline; SDS, sodium dodecyl sulfate; MSP, methylation-specific PCR; MTT, 3-(4,5-dimethylthiazol-2-yl)-2,4-diphenyl-tetrazolium bromide assay; DMSO, dimethyl sulfoxide; OD, optical density

Key words: hepatocellular carcinoma, miR-148a, DNA methylation, DNMT1 that miR-148a acts as a tumor suppressor during hepatocellular carcinogenesis. These results may provide a promising alterative strategy for the therapeutic treatment of HCC.

\section{Introduction}

Primary liver cancer mainly refers to hepatocellular carcinoma (HCC), cholangiocarcinoma and hepatic angiosarcoma. As the third leading cause of cancer death, HCC accounts for 85-90\% of all primary liver cancers and ranks as the fifth most prevalent malignancy worldwide (1). The high mortality of HCC is due to late stage detection, and most of the available therapies are not effective (2). The progression of hepatocellular carcinogenesis is thought to involve the deregulation of genes that are critical to cellular processes, such as cell cycle control, cell growth, apoptosis, cell migration and spreading. In the past few decades, studies have focused on investigating the genes and proteins responsible for the development and progression of HCC (3). Recently, an increasing number of reports have implicated a new class of small regulatory RNA molecules, termed microRNAs (miRNAs), in HCC progression.

Since their discovery in 1993, miRNAs have been described in all multicellular organisms and are associated with a vast breadth of biological functions, including cellular proliferation, cellular differentiation and immunity, as well as tissue remodeling and various human diseases, including cancer (4). A recent demonstration of the differential expression of miRNAs and their target mRNAs in cancer and the discovery that some miRNAs can function as oncogenes or tumor suppressors have sparked considerable interest in elucidating their role in tumorigenesis $(5,6)$. Some specific miRNAs have been found to be frequently deregulated, and this deregulation has been associated with clinicopathological features of HCC, such as metastasis, recurrence and prognosis (7-9). miRNAs are highly conserved, small, non-coding RNAs that negatively regulate gene expression in vertebrates through multiple mechanisms, such as complimentary base pairing with the 3'-UTR of their target mRNAs, resulting in translational repression, mRNA cleavage and mRNA decay initiated by miRNA-guided rapid deadenylation (10). However, recent studies have demonstrated that miRNAs can interact with the 5'-UTR of their target mRNAs and the DNA methylation machinery, thereby affecting chromatin status (11). 
Epigenetic alternations in genomic DNA include cytosine methylation in $\mathrm{CpG}$ islands, which usually extend throughout the promoters and the first exons of genes. DNA methylation, which is associated with gene silencing (12), is carried out by DNA methyltransferases (DNMTs). Recent studies have established that, similar to mutations, methylation-mediated silencing of tumor suppressor genes plays a major role in tumorigenesis. However, unlike mutations, methylation can be reversed by the inhibition of DNA methyltransferase, resulting in restored expression of the silenced tumor suppressor genes. The approval of drugs such as Vidaza ${ }^{\circledR}$ (5-azacytidine) and Dacogen $^{\text {TM }}$ (5-aza-2'-deoxycytidine or decitabine) by the FDA (USA) as anticancer agents underscores the usefulness of epigenetic therapy. Similar to protein-coding genes, DNA sequences encoding miRNAs may undergo aberrant DNA methylation, leading to miRNA upregulation (through DNA hypomethylation) or downregulation (through DNA hypermethylation) in human cancers. For example, previous studies have shown that aberrant hypermethylation of the miR-148a coding region occurs early in human pancreatic carcinogenesis and leads to the downregulation of miR-148a expression (13).

In a previous study, miR-148a was found to be silenced by DNA hypermethylation and to interact with DNMTs in various cancers. Very recently, Gailhouste et al (14) found that miR-148a expression was frequently downregulated in biopsy samples from HCC patients as well as in mouse and human HCC cell lines; however, the mechanism causing this downregulation has not yet been studied in detail. Here, we examine i) whether DNA methylation is involved in the miR148a deregulation that occurs in HCC cell lines; ii) whether there is a circular regulation loop between miR-148a and DNMT1; and iii) the roles that miR-148a plays in the HCC cell cycle. Our study provides new insight into the molecular mechanism of HCC development and yield new strategies for HCC diagnostics and treatment in the future.

\section{Materials and methods}

Cell culture and transient transfection of miR-148a mimics and DNMT1 siRNA. The HCC cell lines HepG2, SMMC-7721 and HCCLM3 and the normal liver cell line L-02 were obtained from Shanghai Fumeng Gene Biological Corporation (Shanghai, China). The cells were maintained in Dulbecco's modified Eagle's medium (DMEM, Gibco, Carlsbad, CA, USA) supplemented with $10 \%$ fetal calf serum (FCS), $100 \mathrm{U} / \mathrm{ml}$ penicillin, $100 \mathrm{mg} / \mathrm{ml}$ streptomycin, and $2 \mathrm{mM} \mathrm{L-glutamine}$ and were incubated at $37^{\circ} \mathrm{C}$ in an atmosphere containing $5 \% \mathrm{CO}_{2}$. On the day of transfection, the HepG2 cells were plated in DMEM supplemented with $10 \%$ FCS at a density of $2-3 \times 10^{5}$ cells $/ \mathrm{ml}$ and were transfected with the miR-148a mimics, DNMT1 siRNA or the non-specific (NS)-miRNA (all at $60 \mathrm{nM}$ ) for $24 \mathrm{~h}$ using Lipofectamine 2000 (Invitrogen) according to the manufacturer's instructions. The culture medium was changed $6 \mathrm{~h}$ after transfection.

The following oligonucleotide sequences were used: miR-148a mimics, 5'-UCAGUGCACUACAGAACUUUGU-3', 5'-AA AGUUCUGUAGUGCACUGAUU-3'; NS-miRNA, 5'-UUCUCCGAACGUGUCACGUTT-3', 5'-ACGUGACACGU UCGGAGAATT-3'; DNMT1 siRNA, 5'-GAGGCCUAUAAU GCAAAGATT-3', 5'-UCUUUGCAUUAUAGGCCUCTT-3'.
One-step quantitative real-time PCR. To confirm the expression of miR-148a, one-step real-time qPCR was performed. Total RNA was extracted from HepG2 using TRIzol reagent (Invitrogen). miR-148a expression was measured using the miScriptII RT kit (Qiagen, Frankfurt, Germany) and the miScript SYBR-Green PCR kit (Qiagen) in an ABI Prism 7500 PCR machine. PCR was performed at $95^{\circ} \mathrm{C}$ for $15 \mathrm{~min}$, followed by 40 cycles of amplification at $94^{\circ} \mathrm{C}$ for $15 \mathrm{sec}$, $55^{\circ} \mathrm{C}$ for $30 \mathrm{sec}$, and $72^{\circ} \mathrm{C}$ for $30 \mathrm{sec}$. The melting curve was performed at $95^{\circ} \mathrm{C}$ for $30 \mathrm{sec}, 60^{\circ} \mathrm{C}$ for $30 \mathrm{~min}$, and $95^{\circ} \mathrm{C}$ for $30 \mathrm{sec}$. The relative miRNA expression was calculated from three different experiments. The fold change of the miRNA relative to the U6 RNA was determined using the formula $2^{-\Delta \Delta C t}$.

Quantitative real-time $P C R$ and semi-quantitative reverse transcription-polymerase chain reaction (RT-PCR). Total RNA was isolated from HepG2, SMMC-7721, HCCLM3 and L02 cells using TRIzol reagent (Invitrogen), and first-strand cDNA was synthesized using the Thermoscript RT-PCR synthesis kit (Fermentas, Pittsburgh, PA, USA) according to the manufacturer's instructions. Quantitative RT-PCR analyses for DNMT1 and GAPDH were performed using the RT-PCR kit (Applied Biosystems, Foster City, CA, USA). The mRNA level of GAPDH was used as an internal control. RT-PCR was carried out according to the standard protocol using the following primers: $\beta$-actin (forward, 5'-TGAGCTGCGTGTGGCCCCTGAG-3'; reverse, 5'-GGGGCATCGGAACCGCTCATTG-3'), DNMT1 (forward, 5'-ACGAGGATGAGAGGGAGGAG-3'; reverse, 5'-GGCACTTTGGTGAGTTGAT-3'). PCR was performed at $94^{\circ} \mathrm{C}$ for $5 \mathrm{~min}$, followed by 30-35 cycles of amplification at $94^{\circ} \mathrm{C}$ for $40 \mathrm{sec}, 56^{\circ} \mathrm{C}$ for $40 \mathrm{sec}$ and $72^{\circ} \mathrm{C}$ for $1 \mathrm{~min}$ using an ABI9700 system. The band intensities were measured using a densitometer, and the results were normalized to the levels of $\beta$-actin. The results were independently repeated at least three times from three different pools of template, while each template pool was extracted from at least eight batches of cells.

5-Aza-2'-deoxycytidine treatment. HepG2 cells were seeded overnight in culture dishes, and 5-aza-2'-deoxycytidine (5-azadC; Sigma-Aldrich, St. Louis, MO, USA) was added. The medium was refreshed every $24 \mathrm{~h}$ until the 48 -h treatment was completed.

Methylation-specific PCR (MS-PCR). The methylation status of the miR-148a promoter region was determined by methylation-specific PCR (MSP) using bisulfite-modified DNA. Genomic DNA was extracted using the QIAamp DNA mini kit (Qiagen). Two primer sets were used to amplify the promoter region of the miR-148a gene that contained several CpG sites; one primer set was specific for the methylated sequence (miR-148a-M: forward, 5'-TGATTCGTTTTATTA TCGGTC-3'; reverse, 5'-AACACTAACGACATCGACG-3'), and the other primer set was specific for the unmethylated sequence (miR-148a-U: forward, 5'-TATGATTTGTTTTAT TATTGGTT-3'; reverse, 5'-AACACTAACAACATCAAC AACC $\left.-3^{\prime}\right)$. The primers used in the present study specifically detect the promoter sequence of the PTEN gene rather than that of the PTEN pseudogene. $\mathrm{M}$ and $\mathrm{U}$ are the PCR products of the methylated and unmethylated alleles, respectively. The PCRs for miR-148a-M and miR-148a-U were carried out in 
$50 \mu \mathrm{l}$ volumes containing 1X PCR buffer (15 mmol/ $1 \mathrm{MgCl}_{2}$ ), $2.5 \mathrm{mmol} / \mathrm{l} \mathrm{dNTP}$ mixture, $10 \mathrm{pM}$ each primer, $4 \mathrm{U}$ HotStarTaq DNA polymerase (Qiagen), and 25-50 ng of bisulfite-modified DNA. Amplification was performed in a thermocycler with the following conditions: $94^{\circ} \mathrm{C}$ for $2 \mathrm{~min}$, followed by 36 cycles of $94^{\circ} \mathrm{C}$ for $30 \mathrm{sec}, 54^{\circ} \mathrm{C}$ or $50^{\circ} \mathrm{C}$ for $30 \mathrm{sec}$, and $72^{\circ} \mathrm{C}$ for $45 \mathrm{sec}$, followed by an extension at $72^{\circ} \mathrm{C}$ for $7 \mathrm{~min}$. The methylation-specific PCRs were performed in duplicate.

Cell proliferation assay. Cell proliferation was determined using the standard 3-(4,5-dimethylthiazol-2-yl)-2,4-diphenyltetrazolium bromide (MTT) assay. Briefly, the cells were seeded at a density of $5 \times 10^{3}$ cells per well in 96-well culture plates and transfected with the miR-148a mimics and negative control as described above. Cell proliferation was assessed after $24 \mathrm{~h}$. After culture, $5 \mathrm{mg} / \mathrm{ml}$ MTT was added and incubated at $37^{\circ} \mathrm{C}$ for an additional $4 \mathrm{~h}$; thereafter, the medium was replaced, and the formazan crystals were dissolved in $150 \mu \mathrm{l}$ of dimethyl sulfoxide (DMSO). The optical density (OD) was determined using a Thermomax microplate reader (Bio-Tek EL, Winooski, VT, USA) at a wavelength of $570 \mathrm{~nm}$. All experiments were performed in triplicate and were repeated at least three times.

Cell cycle analysis. For the cell cycle analysis, we used the Cell Cycle and Apoptosis Analysis kit. (Beyotime, Jiangsu, China). The cells were washed three times with cold PBS and subsequently fixed in $70 \%$ ethanol in PBS at $-20^{\circ} \mathrm{C}$ for $12 \mathrm{~h}$. After fixation, the cells were washed with cold PBS and stained with $0.5 \mathrm{ml}$ of propidium iodide (PI) staining buffer, which contained $200 \mathrm{mg} / \mathrm{ml}$ RNase A and $50 \mu \mathrm{g} / \mathrm{ml} \mathrm{PI}$, at $37^{\circ} \mathrm{C}$ for $30 \mathrm{~min}$ in the dark. Analyses were performed on a BD LSR flow cytometer (BD Biosciences, Franklin Lakes, NJ, USA). The experiments were repeated three times.

Apoptosis analysis. For apoptosis analysis, the number of apoptotic cells was quantified using the Annexin V-FITC Apoptosis Detection Kit (BestBio, Shanghai, China) according to the manufacturer's instructions. Early apoptotic cells were defined as Annexin V-positive, PI-negative cells. Analyses were performed on a BD LSR flow cytometer (BD Biosciences). The experiments were repeated three times.

Western blot analysis. The cells were lysed with RIPA lysis buffer (Beyotime, Haimen, China). Whole extracts were prepared, and the protein concentration was determined using a BCA protein assay kit (Boster, Wuhan, China). Total protein (30 or $50 \mathrm{mg}$ ) from the samples was separated by SDS-PAGE and blotted onto a PVDF membrane (Millipore, Billerica, MA, USA). After blocking, the PVDF membrane were incubated for $1 \mathrm{~h}$ with primary antibodies diluted in TBS/Tween-20 (0.075\%) containing 3\% Marvel. A mouse monoclonal antibody raised against DNMT1 (Santa Cruz Biotechnology, Santa Cruz, CA, USA) and an anti- $\beta$-actin antibody (Santa Cruz Biotechnology) were used at a dilution of 1:600. Horseradish peroxidase-conjugated anti-mouse antibodies were used as the corresponding secondary antibodies. After four washes in TBS/Tween-20, the membranes were developed with distilled water, and the proteins were detected using an enhanced chemiluminescence system (ECL-plus kit, Thermo Scientific, Rockford, IL, USA).
Luciferase reporter assay. We constructed 3'-UTR reporter plasmids for use in the dual luciferase reporter assay. The 3'-UTR segments of the DNMT1 gene containing the miR-148a binding sites were amplified by PCR using KOD-Plus-DNA polymerase (Toyobo, Osaka, Japan) and were cloned into the XhoI/NotI sites downstream of the luciferase reporter gene in the psiCHECK-2-Report vector (Promega, Madison, WI, USA); these constructs were named psiCHECK2-TGF- $\beta 2$ 3'-UTR-wt and psiCHECK-2- $\beta$-catenin 3'-UTR-wt, respectively. For the luciferase assay, HepG 2 cells $\left(5 \times 10^{4}\right.$ cells/well) were cultured in 24-well plates. The cells were then co-transfected with $200 \mathrm{ng}$ of the DNMT1 3'-UTR-wt plasmid or the empty vector plasmid in the presence of $60 \mathrm{nmol}$ of the miR-148a mimics (Gene Pharma, Shanghai, China) using $2.5 \mu \mathrm{l}$ of Lipofectamine 2000 and $100 \mu \mathrm{l}$ of Opti-MEM reduced serum medium (Invitrogen, Carlsbad, CA, USA). After 48 h, the luciferase activities were measured consecutively using the Dual-Luciferase Reporter 1000 Assay System (Promega). Renilla luciferase activity was used to normalize the firefly luciferase activity. All the experiments were performed in triplicate.

Statistical analysis. All results are expressed as the mean $\pm \mathrm{SE}$. Statistical significance was determined using either Student's $\mathrm{t}$-test for comparison between the means or a one-way analysis of variance with a post hoc Dunnett's test. $\mathrm{P}<0.05$ was considered to indicate a statistically significant difference.

\section{Results}

miR-148a is significantly downregulated in hepatocellular carcinoma cell lines. To determine whether miR-148a was silenced in HCC cells, we examined the expression of miR-148a using real-time qPCR in the HCC cell lines HepG2, SMMC-7721, and HCCLM3; the normal liver cell line L-02 was used as a matched control. The results show that miR-148a was significantly downregulated in the HCC cell lines (Fig. 1), especially in the HepG2 cells. Therefore, we chose to perform our subsequent studies using the HepG2 cell line.

Downregulation of miR-148a is due to the hypermethylation of the miR-148a gene promoter region in HCC cells. Methylation of gene promoters often occurs during carcinogenesis, resulting in reduced expression or loss of expression of the methylated gene (15). Previous studies have shown that the genomic DNA sequence spanning the miR-148a gene contains a large amount of $\mathrm{CpG}$-rich regions ( $\mathrm{CpG}$ islands) in the promoter (16). Thus, we hypothesized that DNA methylation is responsible for the downregulation of miR-148a in HCC. To verify this hypothesis, we performed MSP analysis to detect the methylation status of the miR-148a promoter region; indeed, hypermethylation of the $\mathrm{CpG}$ islands in the miR-148a promoter was observed in the HepG2, SMMC7721, and HCCLM3 cells compared to the L-02 cells (Fig. 2A). Additionally, to further support the functional relevance of the DNA methylation, we found that demethylation by 5 -aza-dC dramatically restored miR-148a expression in HepG2 cells, and this response was dose-dependent (Fig. 2C). Thus, the MSP results showed that 5 -aza-dC treatment caused the demethylation of the miR-148a promoter (Fig. 2B). 


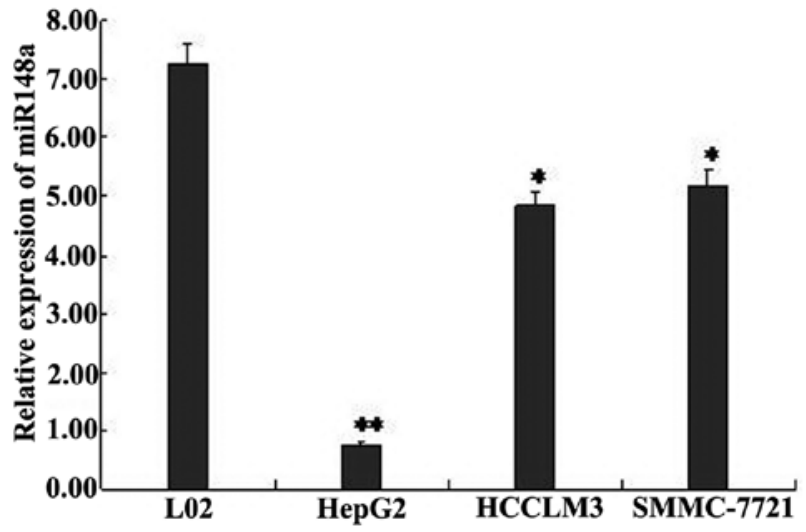

Figure 1. miR-148a is significantly downregulated in the hepatocellular carcinoma cell lines HepG2, HCCLM3 and SMMC-7721 compared to the normal liver cell line L-02. The results are expressed relative to the expression of the control group without treatment. ${ }^{* *} \mathrm{P}<0.01 ;{ }^{*} \mathrm{P}<0.05$ vs. control.

Overexpression of DNMT1 is responsible for hypermethylation of the miR-148a gene promoter in hepatocellular carcinoma cells. Previous studies have shown that DNMT1 overexpression contributes to gene promoter hypermethylation and is associated with the malignant potential and poor prognosis of human cancers $(17,18)$. In our study, we found that DNMT1 expression was strongly increased in the HCC cell lines compared to the L-02 cells (Fig. 3A). To further explore the role of DNMT1 in the regulation of miR-148a expression, we silenced de novo DNMT1 expression using a siRNA targeted against DNMT1. DNMT1 knockdown abolished the hypermethylation of the miR-148a gene (Fig. 3B) and resulted in the upregulation of miR-148a expression (Fig. 3C). These data strongly suggest that overexpression of DNMT1 is responsible for the hypermethylation of the miR-148a gene promoter in HCC cells.

DNMT1 is a direct target of $m i R-148 a$. It has been reported that miR-148a directly targets DNMT1 in lupus CD4 ${ }^{+} \mathrm{T}$ cells and gastric cancer $(19,20)$. The TargetScan 5.1 online software (http://www.targetscan.org/, Whitehead Institute for Biomedical Research, Cambridge, MA, USA) was used to predict the miR-148a target genes, and unsurprisingly, we found that the 3'-UTR of the DNMT1 gene had 7 sequential bases that paired with the 5 ' end of human miR-148a (Fig. 4A). This finding indicates that DNMT1 may be a potential target of miR-148a. Additionally, co-transfection of miR-148a mimics and the DNMT1-wt construct caused a significant decrease in luciferase activity compared to transfection with the NS-miRNA in HepG2 cells (Fig. 4B). Furthermore, western blot and real-time PCR analyses revealed that DNMT1 protein and mRNA expression was significantly lower in the miR-148a-transfected HepG2 cells compared to the control group (Fig. 4C). These results suggest that DNMT1 is a direct target of miR-148a in HCC cells.

miR-148a inhibits cell proliferation. To investigate the roles of miR-148a in the regulation of HCC cell proliferation and apoptosis, we tested the effects of miR-148a on the proliferation of HepG2 cells. We found that the cells transfected with the miR-148a mimics had significantly increased expression of
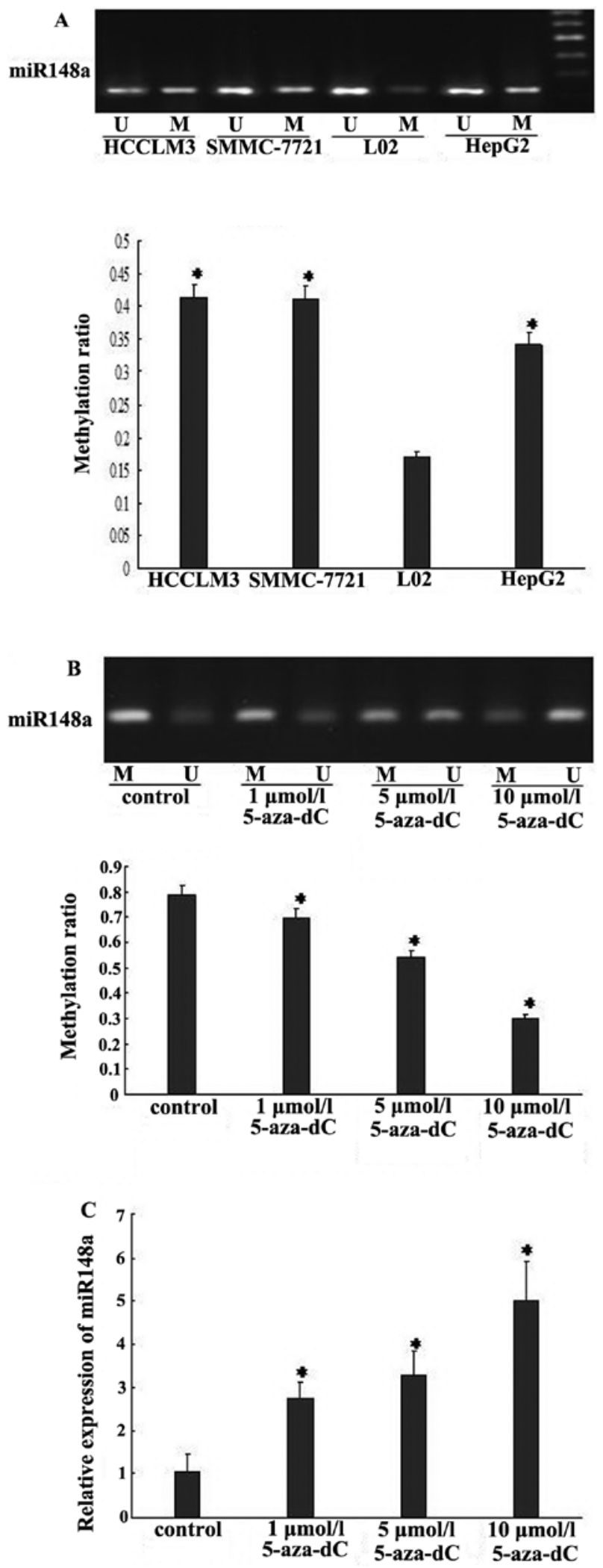

Figure 2. The downregulation of miR-148a is due to the hypermethylation of the miR-148a promoter region in HCC cells. (A) The methylation status of the miR-148a promoter region was detected using MSP. M and U: PCR products of the methylated and unmethylated alleles, respectively. (B) MSP analysis of the miR-148a promoter in HepG2 cells challenged with or without different concentrations of 5 -azadC $(1,5$ or $10 \mu \mathrm{mol} / \mathrm{l})$ for $48 \mathrm{~h}$. (C) One-step quantitative real-time PCR analysis of miR-148a expression in HepG2 cells challenged with or without different concentrations of 5-azadC $(1,5$ or $10 \mu \mathrm{mol} / 1)$ for $48 \mathrm{~h}$. The results are expressed relative to the expression of the control group without treatment. ${ }^{*} \mathrm{P}<0.05$ vs. control. 

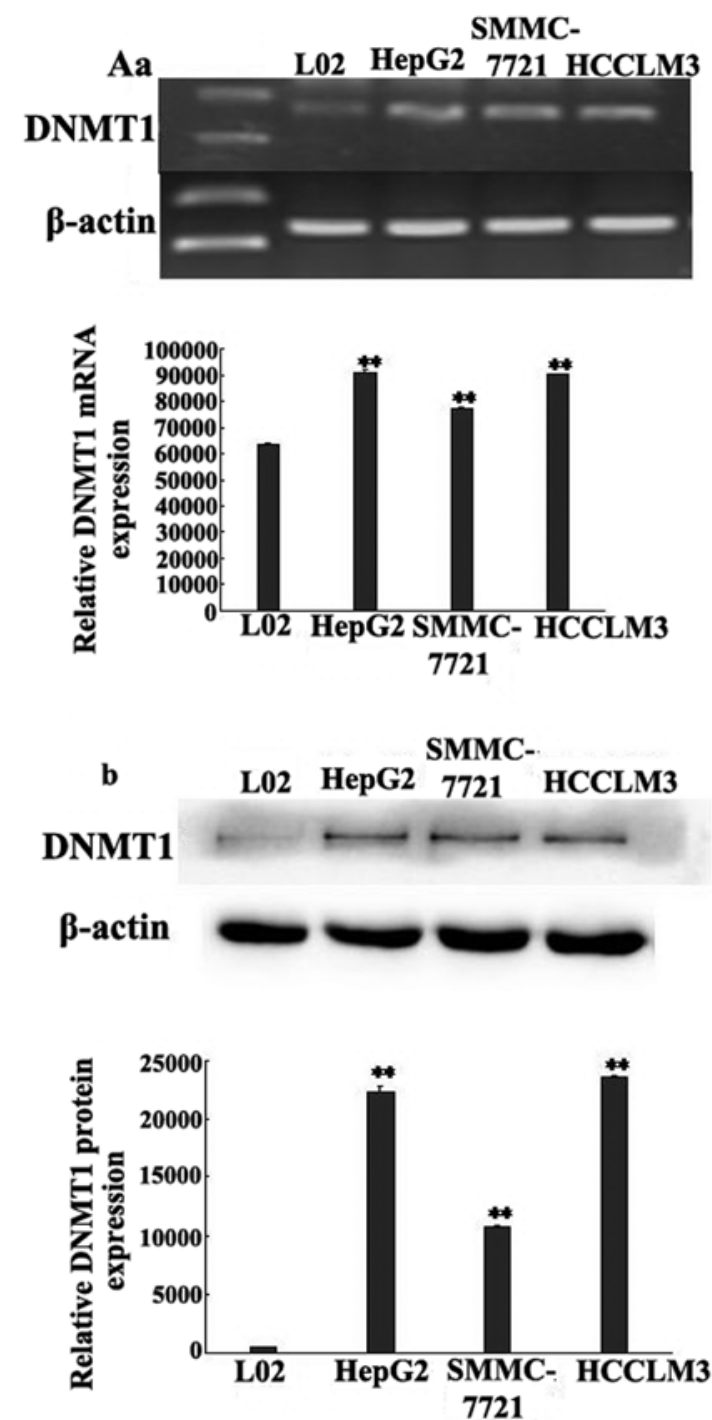
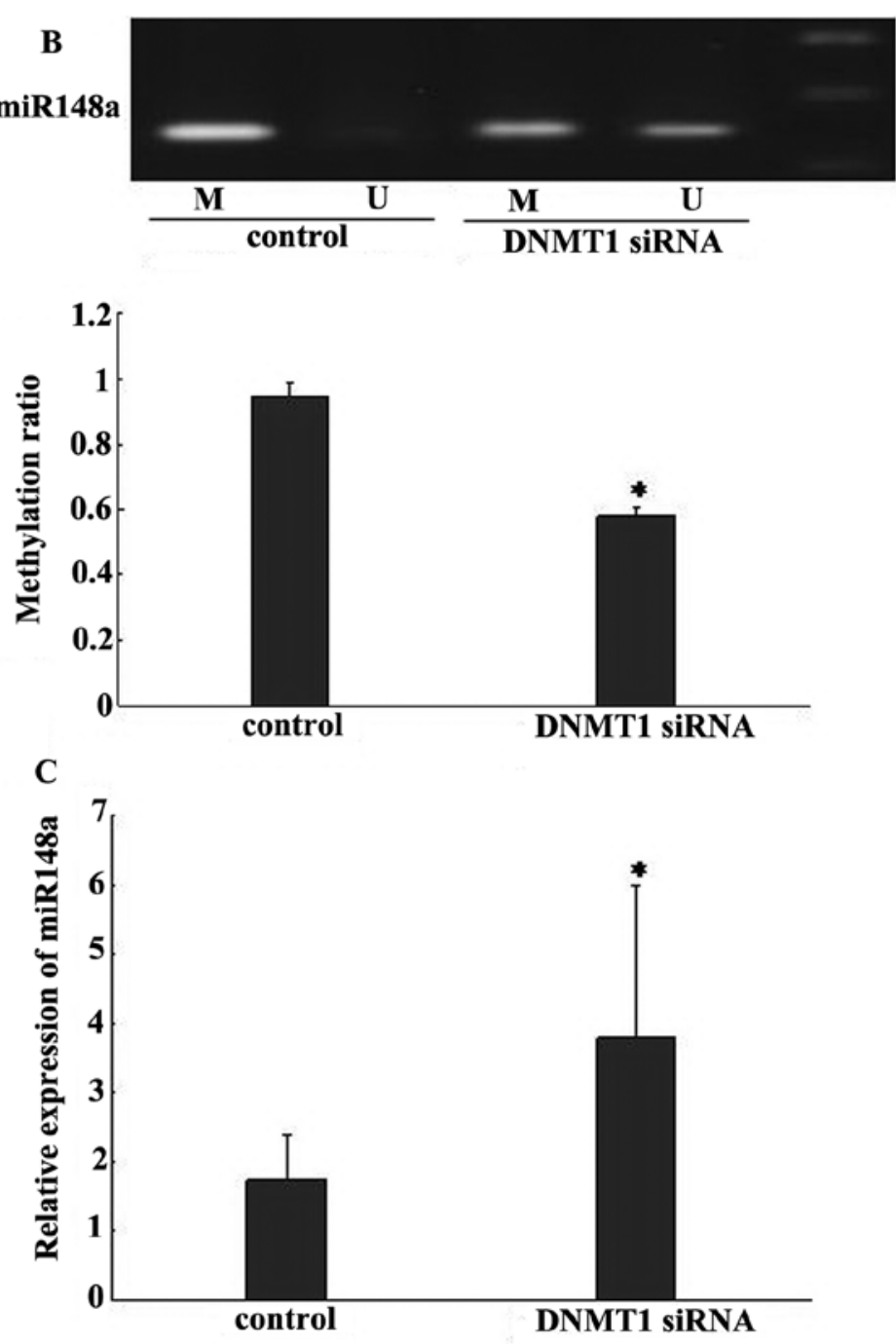

Figure 3. The overexpression of DNMT1 is responsible for the hypermethylation of the miR-148a promoter in HepG2 cells. (A) DNMT1 mRNA and protein levels were analyzed using (a) semi-quantitative reverse transcription-polymerase chain reaction (RT-PCR) and (b) western blot analyses in the L02 cells compared to the HCC cell lines (HepG2, SMMC-7721 and HCCLM3). (B) MSP analysis of the miR-148a promoter in HepG2 cells transfected with or without DNMT1 siRNA for $24 \mathrm{~h}$. (C) One-step quantitative real-time PCR analysis of miR-148a expression from HepG2 cells transfected with or without DNMT1 siRNA for 24 h. The results are expressed relative to the expression of the control group without treatment. ${ }^{* *} \mathrm{P}<0.01 ;{ }^{*} \mathrm{P}<0.05$ vs. control.

mature miR-148a (Fig. 5A). The MTT assay showed that the introduction of miR-148a caused significant inhibition of HCC cell proliferation (Fig. 5B). To understand whether the reduced cell proliferation was due to cell cycle arrest or apoptosis, we used FACS analysis to measure the effect of miR-148a on cell cycle progression and apoptosis. We found that overexpression of miR-148a had a striking effect on cell cycle distribution, whereas the proportion of apoptotic cells induced by the transfection of the miR-148a mimics was not significantly different from that induced by transfection of NS-miRNA (Fig. 5C). These results indicated that overexpression of miR-148a inhibited HCC cell proliferation, at least in part, through cell cycle arrest; however, overexpression did not influence cell apoptosis.

\section{Discussion}

In recent years, there has been increasing interest in the roles of epigenetic modifications in the etiology of human diseases
$(13,21)$. For example, aberrant hypermethylation of the $\mathrm{CpG}$ islands of tumor suppressor genes and the resulting transcriptional silencing are associated with malignant transformation in cancer (22). At the same time, a large number of studies have revealed that microRNAs constitute effective regulatory networks and can regulate approximately one-third of the human protein coding genes at the post-transcriptional level. Some miRNAs are known as gene silencers, and their expression profiles have been negatively correlated with their target genes, including oncogenes, during carcinogenesis. Interestingly, aberrant expression of these miRNAs has been reported in most tumors; thus, miRNAs may play a critical role in tumor formation and development.

Hepatocellular carcinoma (HCC) has an extremely poor prognosis and remains one of the most common and aggressive human malignancies worldwide (23). The effects of epigenetic changes on HCC, especially those related to the biological functions of miRNAs, have been extensively reported in recent 

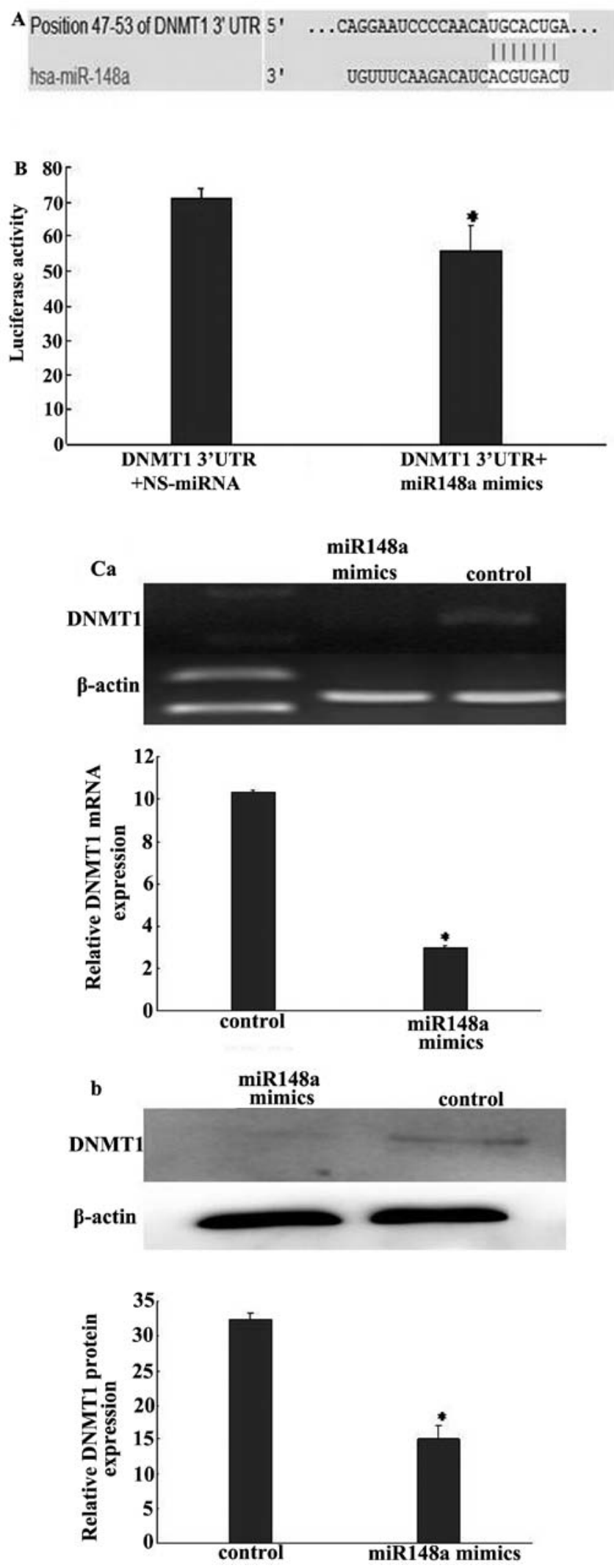

Figure 4. DNMT1 is a direct target of miR-148a. (A) The region of the DNMT1 3'-UTR predicted to be targeted by miR-148a. Three different bioinformatics approaches (miRbase, miRANDA and Targetscan 5.1) were used for the target prediction. (B) Analysis of the luciferase activity. The 3'-UTR of DNMT1 was fused to the firefly luciferase gene and was co-transfected into HepG2 cells with NS-miRNA or the miR-148a mimics. Luciferase activity was assayed $48 \mathrm{~h}$ after transfection. (C) DNMT1 mRNA and protein levels in HepG2 cells transfected with or without the miR-148a mimics were analyzed using (a) real-time PCR and (b) western blot analysis. The results are expressed relative to the expression of the control group. ${ }^{*} \mathrm{P}<0.05$ vs. control. years. Zhang et al (24) found that miR-148a suppresses the epithelial-mesenchymal transition (EMT) and metastasis of hepatoma cells by targeting the Met/Snail signaling pathway. Han et al (25) indicated that Myc induces HCC through a novel, microRNA-mediated feedback loop composed of miR-148a-5p, miR-363-3p and ubiquitin-specific protease 28 (USP28). Moreover, Yan et al (26) found that miR-148a inhibits the metastasis of HCC cells by blocking the EMT and CSC-like properties through the Wnt signaling pathway. In this study, we confirmed the downregulation of miR-148a in HCC cell lines using real-time qPCR and demonstrated that the restoration of miR-148a expression in HCC by transfection with miR-148a mimics could obviously inhibit cell proliferation, suggesting that miR-148a plays a tumor suppressive role in HCC. These findings also have potential therapeutic implications. We found that the methylation level of the $\mathrm{CpG}$ islands in the miR-148a promoter was higher in HCC cells than in normal liver cells, and miR-148a was upregulated in HCC cell lines upon treatment with the DNA hypomethylating agent 5-aza-2-dC. These results indicate that the silencing of miR-148a was caused by the hypermethylation of its promoter region in HCC.

DNA methylation is carried out by the DNMTs, which are ubiquitously expressed in normal human tissues (27). In cancer, they may be overexpressed in various tumor types, such as leukemia, colorectal, ovarian, prostate and breast cancer (28-31). We assayed the expression of DNMT1 in the HCC cell lines compared to the normal liver cells. Additionally, we examined the expression of miR-148a and the methylation level of the miR-148a promoter after siRNA-mediated DNMT1 depletion in HCC cell lines. The results showed that the expression of DNMT1 was remarkably higher in the HCC cells, whereas the methylation level of the miR-148a promoter was significantly reduced, and miR-148a expression was significantly upregulated after DNMT1 knockdown, suggesting that DNMT1 was overexpressed and was responsible for the silencing of miR-148a in HCC. This study is the first to reveal that DNMT1 plays a critical role in regulating miR-148a expression by controlling the methylation level of CpG islands in HCC. The regulation of miR-148a by DNMT1 explains why miR-148a is upregulated after treatment with 5-aza-2-dC or DNMT1 knockdown and suggests an important functional link between DNMT1 and miR-148a.

DNMT1 is a methyltransferase that maintains methylation patterns. Our previous research indicated that DNMT1-mediated PTEN hypermethylation confers hepatic stellate cell activation and liver fibrogenesis in rats (32). Other studies have shown that DNMT1 overexpression contributes to gene promoter hypermethylation and is associated with the malignant potential and poor prognosis of human cancers $(17,18)$. Additionally, Huang et al (33) found that the downregulation of miRNA-152 could induce aberrant DNA methylation in hepatitis B virus-related HCC by targeting DNMT1. In this study, we found that the DNMT1 mRNA and protein levels were repressed after the restoration of miR-148a expression, suggesting that DNMT1 might be one of the targets of miR-148a. This hypothesis was also confirmed using the TargetScan program and luciferase reporter assays. Recent studies in both human cholangiocarcinoma (34) and systemic lupus erythematosus (SLE) (20) demonstrated that DNMT1 was directly regulated by miR-148a. However, these 
A HepG2 cells transfected with miR148a mimics

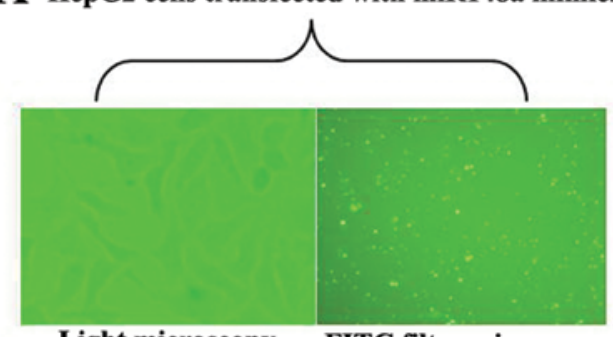

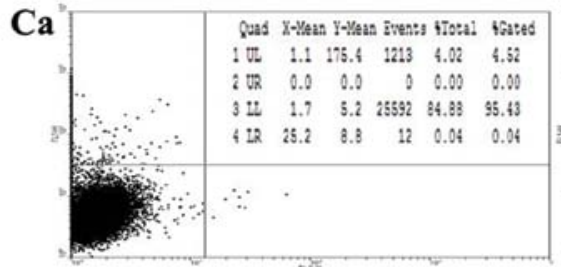

NC-miRNA
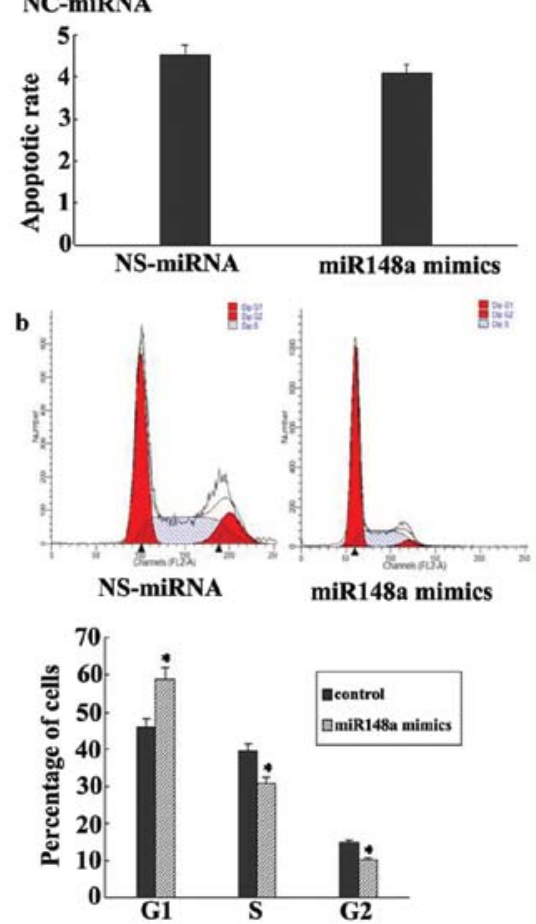

Figure 5. Effect of miR-148a on cell proliferation, cell cycle and apoptosis in HepG2 cells. (A) Upregulation of miR-148a expression in transfected HepG2 cells. Light micrograph of HepG2 cells transfected with fluorescently labeled miR-148a $24 \mathrm{~h}$ after transfection. miR-148a expression in HepG2 cells was analyzed using one-step quantitative real-time PCR. The results are expressed relative to the expression of the control group. ${ }^{* *} \mathrm{P}<0.01 \mathrm{vs}$. NS-miRNA and control. (B) miR-148a overexpression significantly inhibited the proliferation of HepG2 cells. The role of miR-148a in the regulation of HepG2 proliferation was tested using an MTT assay. The data represent the mean \pm SD of three different experiments. ${ }^{*} \mathrm{P}<0.05$ vs. NS-miRNA. (C) Effect of miR-148a on cell cycle progression and apoptosis in HepG2 cells. Apoptosis analysis showed no difference between the HepG2 cells transfected with the miR-148a mimics and those transfected with NS-miRNA (a). However, overexpression of miR-148a significantly affected the cell cycle progression of the HepG2 cells, as shown by the increased proportion of cells in G0/G1 phase and the reduced proportion of cells in S phase (b). Three independent experiments were performed, and one representative experiment is shown.

studies drew different conclusions regarding the way in which miR-148a targets DNMT1; the recognition region was thought to be in either the 3'-UTR (34) or the coding region (20).

Taken together, the silencing of miR-148a and the overexpression of DNMT1 may result in a regulatory feedback loop in HCC. On the one hand, overexpression of DNMT1 leads to the hypermethylation of the miR-148a promoter region, thus causing miR-148a silencing; on the other hand, restoration of miR-148a induces the downregulation of DNMT1. However, in hepatocellular carcinogenesis, the silencing of miR-148a caused by hypermethylation reduces its suppression of DNMT1, resulting in higher DNMT1 expression and the hypermethylation of the miR-148a gene. On the basis of the above results, we came to the following conclusions. First, DNA methylation is involved in the deregulation of miR-148a in HCC. Second, there is a regulatory feedback loop between miR-148a and DNMT1. Third, miR-148a can inhibit cell proliferation via cell cycle arrest but does not influence cell apoptosis. Our study provides new insight into the molecular mechanisms of HCC development and presents potential strategies for HCC diagnostics and treatment in the future.

Very recently, Gailhouste et al (14) found that miR-148a expression was frequently downregulated in biopsy samples from HCC patients as well as in mouse and human HCC cell lines; however, these authors did not focus on the relationship between miR-148a and systemic DNA methylation. Therefore, the impact of this relationship on HCC cells remained unknown until our study was performed. Recent studies have shown that miR-148a suppressed the EMT by targeting ROCK1 in non-small cell lung cancer cells (35) and regulated immune homeostasis by targeting CaMKIIa (36). It is likely that miR-148a may have different functional targets in different types of cancers; this hypothesis requires further investigation. However, a miR-148a knockout mouse has not yet been generated, and the current knowledge of miR-148a functions in HCC is still very limited. Further study using knockout and transgenic animal models will aid in the identification of the in vivo functions of miR-148a in HCC. 
Due to the hypermethylation of its CpG island, miR-148a undergoes methylation-mediated silencing in HCC cell lines. Additionally, DNMT1 is aberrantly upregulated in HCC cell lines, and its overexpression is responsible for hypermethylation of the miR-148a promoter. Interestingly, the expression of DNMT1, which is a target of miR-148a, is inversely correlated with the expression of miR-148a in HCC cells. These results led us to propose a negative feedback regulatory loop between miR-148a and DNMT1 in HCC. Importantly, the overexpression of miR-148a significantly inhibited HCC cell proliferation and cell cycle progression. Our results suggest the existence of a novel miR-148a-DNMT1 regulatory circuit and indicate that miR-148a may act as a tumor suppressor in hepatocellular carcinogenesis.

\section{Acknowledgements}

This project was supported by the National Science Foundation of China (nos. 81072686, 81273526 and 81202978).

\section{References}

1. Farazi PA and DePinho RA: Hepatocellular carcinoma pathogenesis: from genes to environment. Nat Rev Cancer 6: 674-687, 2006.

2. El-Serag HB and Rudolph KL: Hepatocellular carcinoma: epidemiology and molecular carcinogenesis. Gastroenterology 132: 2557-2576, 2007

3. Aravalli RN, Steer CJ and Cressman EN: Molecular mechanisms of hepatocellular carcinoma. Hepatology 48: 2047-2063, 2008.

4. Zhang B, Wang Q and Pan X: MicroRNAs and their regulatory roles in animals and plants. J Cell Physiol 210: 279-289, 2007.

5. Calin GA and Croce CM: MicroRNA signatures in human cancers. Nat Rev Cancer 6: 857-866, 2006.

6. Calin GA and Croce CM: Chromosomal rearrangements and microRNAs: a new cancer link with clinical implications. J Clin Invest 117: 2059-2066, 2007.

7. Braconi $\mathrm{C}$ and Patel T: MicroRNA expression profiling: a molecular tool for defining the phenotype of hepatocellular tumors. Hepatology 47: 1807-1809, 2008.

8. Ladeiro Y, Couchy G, Balabaud C, et al: MicroRNA profiling in hepatocellular tumors is associated with clinical features and oncogene/tumor suppressor gene mutations. Hepatology 47: 1955-1963, 2008.

9. Mott JL: MicroRNAs involved in tumor suppressor and oncogene pathways: implications for hepatobiliary neoplasia. Hepatology 50: 630-637, 2009.

10. Filipowicz W, Bhattacharyya SN and Sonenberg N: Mechanisms of post-transcriptional regulation by microRNAs: are the answers in sight? Nat Rev Genet 9: 102-114, 2008.

11. Qiu L, Fan H, Jin W, et al: miR-122-induced down-regulation of HO-1 negatively affects miR-122-mediated suppression of HBV. Biochem Biophys Res Commun 398: 771-777, 2010.

12. Wolffe AP and Matzke MA: Epigenetics: regulation through repression. Science 286: 481-486, 1999.

13. Hanoun N, Delpu Y, Suriawinata AA, et al: The silencing of microRNA 148a production by DNA hypermethylation is an early event in pancreatic carcinogenesis. Clin Chem 56: 1107-1118, 2010.

14. Gailhouste L, Gomez-Santos L, Hagiwara K, et al: miR-148a plays a pivotal role in the liver by promoting the hepatospecific phenotype and suppressing the invasiveness of transformed cells. Hepatology 58: 1153-1165, 2013.

15. Wilson AS, Power BE and Molloy PL: DNA hypomethylation and human diseases. Biochim Biophys Acta 1775: 138-162, 2007.
16. Xu Q, Jiang Y, Yin Y, et al: A regulatory circuit of miR-148a/152 and DNMT1 in modulating cell transformation and tumor angiogenesis through IGF-IR and IRS1. J Mol Cell Biol 5: 3-13, 2013.

17. Bernardino J, Roux C, Almeida A, et al: DNA hypomethylation in breast cancer: an independent parameter of tumor progression? Cancer Genet Cytogenet 97: 83-89, 1997.

18. Soares J, Pinto AE, Cunha CV, et al: Global DNA hypomethylation in breast carcinoma: correlation with prognostic factors and tumor progression. Cancer 85: 112-118, 1999.

19. Zhu A, Xia J, Zuo J, et al: MicroRNA-148a is silenced by hypermethylation and interacts with DNA methyltransferase 1 in gastric cancer. Med Oncol 29: 2701-2709, 2012.

20. Pan W, Zhu S, Yuan M, et al: MicroRNA-21 and microRNA-148a contribute to DNA hypomethylation in lupus $\mathrm{CD} 4^{+} \mathrm{T}$ cells by directly and indirectly targeting DNA methyltransferase 1 . J Immunol 184: 6773-6781, 2010.

21. Egger G, Liang G, Aparicio A and Jones PA: Epigenetics in human disease and prospects for epigenetic therapy. Nature 429: 457-463, 2004.

22. Balaguer F, Link A, Lozano JJ, et al: Epigenetic silencing of miR-137 is an early event in colorectal carcinogenesis. Cancer Res 70: 6609-6618, 2010.

23. Thorgeirsson SS and Grisham JW: Molecular pathogenesis of human hepatocellular carcinoma. Nat Genet 31: 339-346, 2002.

24. Zhang JP, Zeng C, Xu L, Gong J, Fang JH and Zhuang SM: MicroRNA-148a suppresses the epithelial-mesenchymal transition and metastasis of hepatoma cells by targeting Met/Snail signaling. Oncogene: Sep 9, 2013 (Epub ahead of print).

25. Han H, Sun D, Li W, et al: A c-Myc-MicroRNA functional feedback loop affects hepatocarcinogenesis. Hepatology 57: 2378-2389, 2013.

26. Yan H, Dong X, Zhong X, et al: Inhibitions of epithelial to mesenchymal transition and cancer stem cells-like properties are involved in miR-148a-mediated anti-metastasis of hepatocellular carcinoma. Mol Carcinog: Jul 17, 2013 (Epub ahead of print).

27. Robertson KD, Uzvolgyi E, Liang G, et al: The human DNA methyltransferases (DNMTs) 1, 3a and 3b: coordinate mRNA expression in normal tissues and overexpression in tumors. Nucleic Acids Res 27: 2291-2298, 1999.

28. Ahluwalia A, Hurteau JA, Bigsby RM and Nephew KP: DNA methylation in ovarian cancer. II. Expression of DNA methyltransferases in ovarian cancer cell lines and normal ovarian epithelial cells. Gynecol Oncol 82: 299-304, 2001.

29. Karpf AR and Matsui S: Genetic disruption of cytosine DNA methyltransferase enzymes induces chromosomal instability in human cancer cells. Cancer Res 65: 8635-8639, 2005.

30. Mizuno S, Chijiwa T, Okamura T, et al: Expression of DNA methyltransferases DNMT1, 3A, and 3B in normal hematopoiesis and in acute and chronic myelogenous leukemia. Blood 97: 1172-1179, 2001.

31. Roll JD, Rivenbark AG, Jones WD and Coleman WB: DNMT3b overexpression contributes to a hypermethylator phenotype in human breast cancer cell lines. Mol Cancer 7: 15, 2008.

32. Bian EB, Huang C, Ma TT, et al: DNMT1-mediated PTEN hypermethylation confers hepatic stellate cell activation and liver fibrogenesis in rats. Toxicol Appl Pharmacol 264: 13-22, 2012.

33. Huang J, Wang Y, Guo Y and Sun S: Down-regulated microRNA-152 induces aberrant DNA methylation in hepatitis B virus-related hepatocellular carcinoma by targeting DNA methyltransferase 1. Hepatology 52: 60-70, 2010.

34. Braconi C, Huang N and Patel T: MicroRNA-dependent regulation of DNA methyltransferase-1 and tumor suppressor gene expression by interleukin- 6 in human malignant cholangiocytes. Hepatology 51: 881-890, 2010.

35. Li J, Song Y, Wang Y, Luo J and Yu W: MicroRNA-148a suppresses epithelial-to-mesenchymal transition by targeting ROCK1 in non-small cell lung cancer cells. Mol Cell Biochem 380: 277-282, 2013.

36. Liu X, Zhan Z, Xu L, et al: MicroRNA-148/152 impair innate response and antigen presentation of TLR-triggered dendritic cells by targeting CaMKIIalpha. J Immunol 185: 7244-7251, 2010 . 